\title{
Polyphonie dans le discours journalistique : une étude comparative de la presse anglophone et francophone
}

Lucile Davier

\section{CpenEdition}

Journals

Édition électronique

URL : http://journals.openedition.org/asp/145

DOI : 10.4000/asp. 145

ISBN : 978-2-8218-0410-4

ISSN : 2108-6354

Éditeur

Groupe d'étude et de recherche en anglais de spécialité

\section{Édition imprimée}

Date de publication : 1 novembre 2009

Pagination : $67-88$

ISSN : 1246-8185

\section{Référence électronique}

Lucile Davier, « Polyphonie dans le discours journalistique : une étude comparative de la presse anglophone et francophone », ASp [En ligne], 56 | 2009, mis en ligne le 01 novembre 2012, consulté le 02 novembre 2020. URL : http://journals.openedition.org/asp/145 ; DOI : https://doi.org/10.4000/asp. 145

Ce document a été généré automatiquement le 2 novembre 2020.

Tous droits réservés 


\title{
Polyphonie dans le discours journalistique : une étude comparative de la presse anglophone et francophone
}

\author{
Lucile Davier
}

1 Le discours journalistique est souvent considéré comme un cas limite du discours de spécialité. En effet, quand on pense au grand reportage, ce sont des noms comme Joseph Kessel ou Albert Londres qui viennent à l'esprit, des noms qu'on associe plutôt à une grande tradition littéraire. On sait également que Balzac et Zola ont frayé avec le journalisme. Mais depuis la seconde moitié du XXe siècle, le journalisme tourne le dos à cet héritage pour aller voir du côté de la vulgarisation: il emprunte donc largement aux discours spécialisés sans pour autant verser dans leur jargon.

2 De plus, ce n'est pas l'écriture journalistique à proprement parler, mais bien plus le genre de discours et ses spécificités qui sont le centre d'intérêt de cette étude. La terminologie de Maingueneau (2007) a été choisie pour parler de la presse écrite comme type de discours ${ }^{1}$ et de l'article (ou news en anglais) comme genre de discours. Le genre de discours met en œuvre des dispositifs et des fonctions qui, comme il en sera question plus tard, varient d'une communauté culturelle et linguistique à une autre ${ }^{2}$. Ces variations sont particulièrement notables dans le domaine de la polyphonie ${ }^{3}$ : citations au discours direct ou indirect, rapport de certitude à des discours tiers, mémoire collective, implicites culturels, etc. (Maingueneau 2007 : 110-121). Sophie Moirand généralise ce constat dans des termes très imagés :

Le texte journalistique devient alors une mosaïque de voix, constituée d'une pluralité de fils intertextuels, et le fil horizontal du discours apparait, dans sa matérialité même, fracturé par des marques de cette hétérogénéité [...]. (2007 : 85)

3 C'est dans cette perspective que l'approche comparativiste - ici entre l'anglais et le français - paraît toute désignée pour mettre en relief des phénomènes qui passeraient inaperçus au sein d'une seule langue. On tentera donc de répondre à la question 
suivante : la polyphonie du discours journalistique se traduit-elle différemment dans la presse quotidienne francophone et anglophone? Pour ce faire, il sera nécessaire de présenter la méthode (l'analyse de contenu et l'analyse de discours) et le corpus de ce travail (constitué à la fois d'une sélection d'articles de presse et des discours officiels d'où les journalistes tirent la majeure partie de leurs informations) avant d'aborder les deux axes d'analyse: la polyphonie et les pratiques journalistiques. Dans la partie portant sur la polyphonie sont d'abord examinés les rapports qu'entretiennent les articles avec leurs sources principales (appelés « discours-source » dans le corpus), puis les traces d'une mémoire collective occidentale en lien avec le terrorisme islamiste et enfin les grandes lignes du cadre d'interprétation qui domine les différents articles du corpus. La seconde partie explore au contraire les différences de représentation qui peuvent apparaître dans les articles du corpus en raison premièrement des particularités sociales, historiques ou politiques de chaque nation; deuxièmement des formations et des pratiques journalistiques divergeant d'un pays à l'autre; troisièmement des interprétations nationales de l'événement terroriste. Une réflexion sur l'exploitation possible des résultats obtenus est amorcée en guise de conclusion.

\section{Méthodologie et corpus}

\subsection{Méthodologie}

4 Travaillant dans le domaine des médias, il semble impossible de se cantonner à des outils traditionnellement utilisés en analyse littéraire ou en critique de la traduction. C'est pourquoi des instruments adaptés au discours médiatique ont été empruntés aux sciences de la communication : l'analyse de contenu et l'analyse de discours ${ }^{4}$.

5 L'analyse de contenu, méthode exclusivement quantitative, permet de concrétiser ou de vérifier les intuitions du chercheur. Bardin (1977) et de Bonville (2000) proposent de procéder à une " lecture flottante » - phase préparatrice pendant laquelle on repère les grandes tendances du corpus. Le chercheur établit ensuite des catégories d'analyse (grammaticales, thématiques et sémantiques) dont il comptabilise les occurrences. À titre d'exemple, on a calculé la fréquence d'apparition du substantif «terroriste » ou des formes verbales au conditionnel selon le pays, le type de journal, la date, etc. La liste complète des catégories et des sous-catégories est disponible en annexe 1. Le choix des catégories dépend non seulement du chercheur, mais surtout de sa problématique de travail. Ainsi, deux chercheurs peuvent aborder un même corpus sous des angles complètement différents. En revanche, la subjectivité des catégories est limitée par son applicabilité :

En l'occurrence, pour être objectif, le procédé analytique doit être conçu de telle manière que des chercheurs qui répéteraient l'analyse dans les mêmes conditions parviendraient à des résultats semblables. (de Bonville 2000 : 11)

L'analyse de contenu est un travail quasi statistique, qui a porté dans la présente étude sur un nombre de textes plus élevé que l'analyse de discours (environ une centaine) pour assurer une certaine vérifiabilité des résultats. Selon de Bonville, cette méthode se distingue de l'analyse de discours pour la raison suivante :

L'analyse de contenu exploite des systèmes catégoriels spécifiques parce qu'elle aborde le texte à un niveau de généralité auquel les concepts linguistiques ne sont pas opérants ; [...]. (de Bonville $2000: 31$ ) 
7 Enfin, les résultats de l'analyse de contenu ont surtout servi de base pour développer les hypothèses qui sous-tendent l'analyse de discours.

Cette analyse de discours est d'ailleurs un peu particulière, puisqu'elle a été réalisée en parallèle sur des textes en deux langues différentes. Les textes n'ont pas été pris un par un, " en soi ", mais dans leur globalité et leurs ressemblances, car il ne s'agit pas de dégager la structure de chaque article, mais de voir quels fils intertextuels se tissent entre eux. Dans ce but, les réflexions de Maingueneau (2007) sur la polyphonie, le concept de paradigme désignationnel (Moirand 2007) et l'analyse de la mise en mots du terrorisme par Koren (1996) entre autres sont mis à contribution pour cerner les particularités du corpus.

Pour clore cette présentation des outils, il est certes important de préciser que les indices trouvés grâce à ces deux méthodes d'analyse livrent des informations sur les conditions de production (par les journalistes) et de réception (par les lecteurs) des différents articles du corpus, mais que ce travail se limite à une analyse du message (excluant par conséquent toute étude consacrée à la production ou à la réception). Il serait donc illusoire, voire épistémologiquement incorrect, de prétendre tirer de ces observations des conclusions fiables sur les auteurs ou sur les destinataires.

\subsection{Corpus}

Les deux instruments d'analyse présentés en partie 1.1. ont été appliqués à un corpus non traditionnel, c'est-à-dire composé de " two or more monolingual corpora built up on the basis of similar design criteria» (Kenny 2001: 52). Contrairement aux pratiques communes en traductologie, il ne s'agit pas d'un corpus "parallèle », c'est-à-dire constitué de textes-source et de leurs traductions (Kenny 2001). L'auteur de cette typologie émet d'ailleurs de forts doutes quant à l'utilité des corpus comparables bilingues (ou multilingues) :

[...] based on the erroneous assumption that there is a natural way of saying anything in any language and that all we need to do is to find out how to say something naturally in La and Lb. (Kenny $2001: 52$ )

11 C'est pourtant l'approche qu'ont retenue les chercheurs du CEDISCOR ${ }^{5}$, qui procèdent à une analyse de discours en plusieurs langues sur un genre tel que l'interview. Néanmoins, comment mieux étudier la mise en pratique de genres textuels que par l'observation comparative de textes rédigés par des auteurs connaissant intimement les règles implicites ou explicites de ce genre dans leur langue et leur culture? Le corpus de l'étude est unifié par des critères thématiques, linguistiques, médiatiques et temporels. Il englobe des articles qui couvrent un événement médiatique ${ }^{6}$ bien précis : le présumé complot terroriste d'août 2006 qui avait probablement pour cible des avions de ligne assurant la liaison entre Londres et les États-Unis. Cet événement a pour caractéristique principale le fait assez surprenant qu'il n'a pas eu lieu puisque les présumés terroristes ont été arrêtés avant même de monter à bord des avions visés. De plus, la législation britannique protégeant les personnes inculpées (Contempt of Court, cf. partie 3.2.), les journalistes n'ont pas eu accès aux dossiers des suspects. Leurs sources d'information se résument donc presque exclusivement à des déclarations officielles.

La sélection des quotidiens impliquait d'inclure aussi bien des journaux de référence que des journaux populaires ${ }^{7}$. La recherche porte sur plusieurs pays anglophones et 
francophones, c'est pourquoi les titres suivants ont été retenus : l'International Herald Tribune pour les États-Unis ${ }^{8}$; The Guardian et The Sun pour le Royaume-Uni, Le Monde en France ; et en Suisse romande, le quotidien populaire Le Matin, dont on ne trouve plus d'équivalent français en termes de diffusion. Certes, d'autres titres étaient inclus dans l'analyse de contenu (cf. annexe 2), parmi eux : Libération, Le Figaro, Le Temps ${ }^{9}$, La Tribune de Genève ${ }^{10}$. Toutefois, pour rendre le corpus utilisable pour une analyse de discours, il a fallu le circonscrire à des limites temporelles précises. Seuls ont été pris en compte les articles parus entre l'annonce de la nouvelle (le 10 août 2006 pour les premiers) et le 15 août, soit un total de 46 articles (cf. annexe 4). En effet, les occurrences dans la presse deviennent plus sporadiques après la date-butoir du 15 août (cf. annexe 2).

Les conférences de presse et autres discours évoqués précédemment ont été insérés dans le corpus pour des raisons simples : c'est presque exclusivement dans ces discours, abondamment relayés par les agences de presse, que les journalistes ont recueilli les informations nécessaires à la rédaction de leurs articles. Tous ces discours ont été prononcés le 10 août 2006 par les personnalités suivantes : Michael Chertoff (Secrétaire d'État à la sécurité intérieure des États-Unis), Alberto Gonzales (Attorney General des États-Unis), John Reid (Secrétaire d'État à l'Intérieur du Royaume-Uni), Robert Mueller (directeur du FBI), Paul Stephenson (Commissaire adjoint de la Police métropolitaine de Londres, New Scotland Yard) et Peter Clarke (assistant du Commissaire adjoint, chef de l'unité antiterroriste). Ils servent d'une part de textes témoin par rapport au contenu des articles du corpus et d'autre part de point de repère pour suivre le cheminement de l'information. Il n'est pas inutile de se demander comment chaque journal (ou journaliste) a repris cette information et l'a représentée. Cet événement crée une situation toute particulière puisqu'une des variables habituelles - les sources - devient dans le contexte du corpus une donnée fixe. Les outils présentés ci-avant ont tout d'abord permis d'entrer de plain-pied dans la problématique de la polyphonie avec un regard porté particulièrement sur les similitudes de traitement de l'information dans les articles du corpus.

\section{Polyphonie et similitudes}

Dans un premier temps, on peut se demander dans quelle mesure la spécificité des sources réduit la distance interculturelle qui aurait pu séparer ces textes. Ensuite se pose la question d'un imaginaire occidental qui pourrait être partagé par les journaux de notre corpus, puis du cadrage induit par ces valeurs partagées.

\subsection{Le rapport aux discours-source}

15 En premier lieu, l'uniformité des sources crée une importante proximité en matière de références. Il est donc très peu étonnant que les catégories d'acteurs citées le plus souvent soient les hommes politiques (qu'ils soient britanniques ou américains) et les professionnels des services de renseignement. Les hommes politiques jouissent en général d'une forte autorité institutionnelle : désignés par le peuple pour assumer une mission démocratique, leur parole officielle est difficile à mettre en doute. On connaît certes un certain nombre d'affaires où la classe politique a menti au peuple ${ }^{11}$; néanmoins, les déclarations officielles restent une source à laquelle on accorde du crédit. Cette catégorie d'acteurs est complétée par les porte-parole des services secrets. Même si l'on peut supposer une certaine collusion avec le monde politique, les services 
secrets bénéficient d'une double source de légitimité, non seulement par qu'ils sont très proches de l'enquête, mais encore parce qu'ils font l'objet d'une véritable légende (si l'on pense au nombre de séries, de films et de livres dont ils sont le thème principal). Il n'est nullement surprenant que les sources non identifiées arrivent en troisième position dans le corpus étant donné la sensibilité du sujet. Pour rappel, des lois sur la protection des informations antiterroristes sont entrées en vigueur récemment dans les pays concernés (que ce soit le Patriot Act aux États-Unis, les différents Terrorism Acts au Royaume-Uni ou la Loi relative à la lutte contre le terrorisme en France). Le phénomène semble toutefois avoir un écho dans les pratiques de la presse en général si l'on en croit les mots de Richard Keeble (2007: 58) : « the culture of anonymous sourcing will spread ». Cette tendance générale serait donc amplifiée dans le cas particulier de ce corpus. La figure médiatique de l'« expert » se détache également d'après l'analyse des citations. Figure d'habitude incontournable dans tous les types de médias, il apparaît en fait assez peu dans les articles du corpus, toutes langues et tous supports confondus. Ici en effet, les spécialistes du terrorisme s'effacent derrière les enquêteurs. La " mosaïque des voix » (Moirand 2007 : 85) qui résonnent dans ces textes est assez homogène, étant donné la faible variété des sources. Il importe d'abord dans cette partie d'en repérer les points communs pour mieux observer, plus tard, des différences induites par d'autres variables.

Autre similitude découlant de la particularité des sources : le recours à la narration. Si l'on résume la tâche du journaliste aux six W exposés dans la plupart des manuels, on le place face à une situation inextricable à cause du lourd secret qui entoure l'enquête et de l'absence de sources de première main. En effet, on ignore ce qui aurait pu se passer et l'envergure d'éventuels dégâts (What?). On ne peut que spéculer sur l'identité des présumés terroristes puisqu'aucune information officielle n'est délivrée à ce sujet (Who ?). Les cibles potentielles restent tout aussi spéculatives puisque les porte-parole des services secrets ou des gouvernements ne souhaitent pas indiquer le nom des compagnies aériennes visées (Where?). La question du «quand» (When?) est une donnée complètement ignorée. Le "pourquoi " (Why?) est résolu grâce au cadre du terrorisme. Pour terminer, les moyens que les terroristes prévoyaient d'utiliser (hoW ?) sont évoqués de manière on ne peut plus elliptique dans les discours-source. Le matériau informationnel à disposition est peu abondant. Face à ce «non-fait » monté en événement médiatique, les journalistes n'ont guère d'autre choix que de combler les vides laissés par les discours officiels. C'est pourquoi ils puisent dans la palette des ressources linguistiques telles que les constructions hypothétiques et conditionnelles des ressources qui peuvent varier selon la langue et la position du journal.

\subsection{La mémoire collective}

17 En deuxième lieu, l'hypothèse d'une mémoire partagée par le monde occidental - dans le cas du corpus - s'appuie essentiellement sur un spécialiste en communication (Dayan 2006) et sur une théoricienne de l'analyse de discours (Moirand 2007) :

Les médias [...] contribuent eux-mêmes à construire des liens entre ces faits de société et à tisser des fils interdiscursifs entre les dires des différentes communautés concernées par ces événements. (2007:2)

18 Dans le cadre de la présente étude, cette phrase peut être comprise de la façon suivante : le terrorisme islamiste étant principalement représenté comme un risque pour le monde occidental, des «fils interdiscursifs" sont tendus entre les 
communautés culturelles et linguistiques menacées (ou se sentant menacées) par des attentats. Pour être encore plus précis, il est possible de faire appel à la notion de «moment discursif » introduite aussi par Sophie Moirand (2007: 4), c'est-à-dire un événement d'une ampleur médiatique telle qu'il a des répercussions à long terme sur des discours ultérieurs et à propos d'autres événements.

Dans le corpus par exemple, c'est la date du 11 septembre 2001 qui se répète comme un leitmotiv. Le 11 septembre semble être plus qu'une date : il entraîne avec lui tout un cadre interprétatif. Daniel Dayan va également dans ce sens quand il parle de cet attentat meurtrier dans les mots suivants:

Il s'agit de souligner qu'il constitue non pas l'événement mais son prélude ; qu'il est le premier acte d'une dramaturgie qui va s'étendre sur plusieurs mois, voire sur plusieurs années. (2006:7)

Le moment discursif devient donc une référence qui tient lieu d'explication, qui permet d'économiser tout un discours sur les origines et les conséquences d'une situation. Le concept de «famille d'événements » (Moirand 2007 : 7) est étroitement lié à celui de moment discursif : "Cet événement semblait en revanche s'inscrire dans une suite d'événements de même type, une même 'famille' [...]». Sur la base de ce cadre théorique, on peut comprendre le 11 septembre comme l'événement fondateur d'une famille qu'on pourrait nommer " terrorisme islamiste ». On lit alors le complot d'août 2006 à la lumière des attentats du 11 septembre et de ceux qui les ont suivis. La famille d'événements contient non seulement des éléments descriptifs (par exemple la méthode des attentats-suicides, la référence à la religion), mais encore des éléments explicatifs (par exemple l'embrigadement idéologique des terroristes, le lien à une organisation secrète mondiale, etc.). En d'autres termes, les attentats déjoués d'août 2006 n'auraient pas pu être traités de la même manière sans les précédents médiatiques que sont entre autres les attentats du 11 septembre à New York, du 11 mars 2003 à Madrid ou du 7 juillet 2005 à Londres. Néanmoins, si la référence est omniprésente dans tous les articles, elle l'est semble-t-il à différents degrés.

Les autres événements de la même famille sont souvent évoqués en termes comparatifs. Les journalistes les situent parfois simplement dans un rapport temporel (un an, cinq ans après, par exemple). Ils comparent aussi l'ampleur qu'auraient pu prendre les tentatives d'attentats sur des avions transatlantiques («cela aurait été pire que le 11 septembre 2001 » [Mde 11-8-06 ], « the atrocities, which would have eclipsed 9/11 [...] » [Sun 11-8-06]), les méthodes utilisées ( US security officials believe their plot was a direct copy of the Bojinka operation in the mid-90s to bring down 12 western airlines simultaneously over the Pacific Ocean. » [Guard 11-8-06: 1]) ou font appel à d'anciennes interprétations («Leur profil serait donc similaire à celui des auteurs des attentats du 7 juillet 2005. » [Mde 12-8-06]).

De surcroît, dans les articles du corpus, on assiste à la constitution de ce que Moirand appelle un "paradigme désignationnel ", c'est-à-dire l'« ensemble des syntagmes qui renvoient à un concept dans un discours donné » (2007 : 23). Ici, la tentative d'attentats est désignée principalement par les termes de "plot» ou de "conspiracy» dans les discours-source, puis transcrite en français par les synonymes "complot» et " conspiration ». Il est difficile de ne pas penser aux théories du complot en circulation dans les médias, ces schémas simplificateurs qui réduisent une situation complexe à un nombre déterminé d'acteurs et d'enjeux : "Le schéma explicatif rassurant consiste à désigner les responsables de tous nos maux» (Taguieff 2006: 19). En effet, en 
définissant cette tentative d'attentats comme un complot islamiste, les auteurs des articles désignent al-Qaïda comme le grand coupable et tentent de résoudre de manière simpliste un problème en réalité infiniment plus complexe.

\subsection{Le cadrage}

24 En dernier lieu, la notion de cadrage (ou framing en anglais) aide à approfondir la réflexion entamée sur la construction d'une mémoire partagée par plusieurs communautés culturelles et linguistiques qui sont (ou se sentent) confrontées à une même menace. David Weaver présente d'ailleurs une définition du framing lancée par son père fondateur, Entman, en 1993 : «to promote a particular problem definition, causal interpretation, moral evaluation, and/or treatment recommendation for the item described" (cité par Weaver 2007 : 143). Si l'on considère les caractéristiques, ou sèmes, de la famille d'événements dans laquelle s'inscrivent les attentats déjoués de Londres, on peut d'abord dégager une même cible: l'occident, c'est-à-dire le lectorat-cible des quotidiens. Ensuite, le lieu de l'attentat a beau être impossible à prévoir, c'est souvent le même : les avions. En plein processus de mondialisation, c'est un moyen de transport que tout le monde pourrait utiliser. On peut repérer un autre sème fortement symbolique, l'« ennemi intérieur ", pour faire allusion à un concept bien connu de McCarthy ${ }^{12}$.

Dans les articles du corpus, à peu d'exceptions près, les suspects sont représentés comme des Pakistanais de nationalité britannique et comme des immigrés bien intégrés :

Le profil des terroristes présumés : tous sont des citoyens britanniques [...]. Tous sont âgés de 17 à 35 ans, avec une moyenne d'âge de 25 ans. Ils portent des patronymes à consonance d'Asie du Sud [...]. [Fig 11-8-06]

These people were born and brought up in the United Kingdom. Some of them may have parents who were immigrants from Pakistan. [IHT, 11-8-06]

"Oliver is a just an ordinary family man who is expecting a new baby," said a relative. [Guard 11-8-06:2]

En revanche, les discours-source n'évoquent nullement cet aspect des choses, puisqu'ils se refusent à livrer l'identité des personnes arrêtées. Il parait difficile d'expliquer cette ressemblance entre les différents quotidiens autrement que par la diffusion d'une rumeur qui correspondrait en tous points à un manque à combler dans l'imaginaire collectif. Cette idée diffuse de "l'ennemi est parmi nous» s'accorde d'ailleurs parfaitement avec les images véhiculées par les mots " complot» et " conspiration »: un groupe secret infiltré dans les sociétés occidentales chercherait à leur nuire par une subtile organisation. Cette représentation collective peut éventuellement rencontrer un écho dans les peurs du public.

Le cadrage de l'événement dans la perspective de la guerre contre le terrorisme et des attaques du 11 septembre implique forcément une désignation des coupables. D'après Roselyne Koren (1996: 197), dans le cas d'une crise médiatique, c'est l'étape qui suit l'exposition des faits. Le public supporte difficilement le vide, l'absence de coupable. Pourtant, les accusations ne sont pas toujours directes. Pour Koren, la simple utilisation d'un mot peut être accusatrice: "L'argumentation implicite, inscrite dans les dénominations, criminalise le plus souvent le 'terroriste' [...]» (1996: 206). Le mot revient effectivement dans les articles de toutes les zones culturelles étudiées. Dans les textes examinés, les références à des militants islamistes, voire à l'islam, sont 
nombreuses. L'accusation n'est pas toujours lancée, mais le contexte peut être favorable à des associations d'idées qui ne seraient pas suggérées explicitement par les auteurs. Par exemple, quand un journaliste aborde les problèmes que pose la communauté musulmane en Grande-Bretagne en marge de son article sur les tentatives d'attentats, il ne fait que juxtaposer des éléments sans lien apparent, mais l'esprit du lecteur peut les mettre en relation par paresse. De même, dans l'évocation vague de l'origine ethnique des suspects, on retrouve l'une des plus grandes communautés d'immigrants du Royaume-Uni : les Pakistanais. La pointe est lancée et elle correspond probablement aux attentes d'un public britannique. D'une manière générale encore, le stéréotype est complété par la nationalité britannique qu'on attribue aux présumés terroristes. Le portrait-robot dressé est d'une simplicité déconcertante et ne correspond pas tout à fait à la complexité de la situation.

En fin de compte, cette étude comparative dévoile une quantité de similitudes dans la représentation de l'événement terroriste, notamment en raison de sources de seconde main très limitées et d'une mémoire collective fortement influencée par les images et les discours qui ont suivi le 11 septembre dans les pays occidentaux. Ces résultats fournissent déjà quelques pistes pour approfondir les éventuelles divergences de la mise en récit journalistique.

\section{Pratiques journalistiques et implicites culturels divergents}

29 Si l'analyse de contenu laissait déjà deviner quelques différences, l'analyse de discours les a confirmées. La plupart du temps des disparités se cachent en effet derrière une homogénéité de surface. Le cadre théorique, qui emprunte des éléments à l'histoire et à la géopolitique, à l'enseignement du journalisme et un peu à la linguistique, suggère différentes hypothèses.

\subsection{Le contexte historico-politique}

Selon Maurice Halbwachs (1950), les individus peuvent avoir des réactions et des comportements différents selon le groupe national dans lequel ils ont vécu et selon l'histoire de ce groupe national. Partant de cette affirmation, il semble pertinent de rassembler quelques informations sur l'histoire des groupes nationaux étudiés. Il est sans doute préférable d'annoncer que cet aperçu extrêmement bref n'a aucune aspiration de nature historique et qu'il vise simplement à résumer dans leurs plus grandes lignes quelques événements qui ont pu traumatiser les pays où sont basés les quotidiens de notre corpus.

La lecture d'un ouvrage de psychologie comparée (Rachman 1990) suggère de commencer ce survol à la Seconde Guerre mondiale. Rachman compare en effet les réactions de plusieurs peuples européens face aux bombardements de l'armée allemande, et parmi eux, des Britanniques et des Français. Au début de la guerre, les bombardements sur les villes françaises ont créé un mouvement de panique dans la population, qui s'est retrouvée sur les routes de France avec ses possessions. Nombreux sont justement ceux qui ont péri pendant cet exode. Lors des bombardements de Londres par l'aviation allemande, en revanche, Rachman rapporte qu'on a observé dans la population un calme extraordinaire et des réactions tout à fait mesurées. Sans verser 
dans la psychologie des peuples, on peut postuler que cet épisode historique a laissé des traces dans l'inconscient collectif des deux pays et tester l'hypothèse d'une réaction plus pondérée dans la presse britannique. Pour terminer avec cette période, on peut simplement ajouter que la Suisse aussi bien que les États-Unis n'ont pas subi d'attaque directe de leur territoire ${ }^{13}$.

Les expériences de ces pays avec le terrorisme avant la date du 11 septembre 2001 sont elles aussi extrêmement variées. Tandis que la France était aux prises avec les nationalismes basque et corse, la Grande-Bretagne était confrontée à un conflit grave avec l'IRA en Irlande du Nord. La France a également été frappée en 1995 par une vague d'attentats islamistes dans le métro et le TGV. En 1993, les États-Unis ont subi un attentat mineur au World Trade Center attribué à des extrémistes islamistes (six morts, mais un millier de blessés), puis l'attentat d'Oklahoma City en 1995, un attentat revendiqué par l'extrême droite américaine. Pour un certain nombre d'auteurs, le 11 septembre 2001 a marqué une réelle rupture. D'après Wagner-Pacifici, les attentats de New York sont un événement totalement inédit, car les Américains vivaient « dans l'illusion d'une perpétuelle innocence politique » $(2006: 213)$. Elle écrit qu'ils ont vécu cette attaque comme la perte de leur invulnérabilité territoriale. Elle ajoute encore que l'opinion publique a été profondément marquée par la nature imprévue des armes et des cibles. Le traumatisme est d'autant plus fort que sa charge symbolique est lourde. Peu après, on a parlé d'un seul attentat manqué visant la France : une tentative de faire exploser en vol un avion reliant Paris à Miami en décembre 2001. Le Royaume-Uni, en revanche, a été à plusieurs reprises la cible de terroristes, avec un attentat meurtrier dans le métro londonien en juillet 2005, puis des tentatives d'attentat en 2006 et en 2007. Il va de soi que l'on ne considérera pas une tentative d'attentat de la même manière si l'on a été marqué dans sa chair par une attaque terroriste. Cela explique peut-être pourquoi les Britanniques font nettement plus de références au 7 juillet 2005 dans leurs articles, et des références assez allusives, qui devraient être explicitées lors d'une traduction vers le français. Bien que sommaire, ce résumé du passé traumatique des pays étudiés ne peut être clos sans une précision: malgré l'importance qu'accordent les sociétés occidentales au terrorisme islamiste, ce sont avant tout les peuples du Proche-Orient, du Moyen-Orient et du Maghreb qui en souffrent et déplorent le plus de victimes.

\subsection{Les particularités des pratiques journalistiques}

Après ces brèves considérations géopolitiques se posent des questions plus pratiques, liées à l'enseignement et à la pratique de la presse dans les pays pris en compte dans le corpus. Bien entendu, les différences qui pourraient être repérées à ce niveau ne seraient pas des réponses aux résultats de l'analyse, mais plutôt des indices pour orienter la recherche. Des comparaisons ont été menées au niveau macrostructurel (fonctionnement de la presse dans son ensemble), puis au niveau microstructurel (pratique de l'écriture, et notamment des genres journalistiques). Toutes les remarques lues dans des ouvrages généraux (tels que Glaser 1998, Bertrand 1995 et Bertrand 1998) reviennent sur l'opposition théorique entre facts et comments, qui ne serait pas appliquée de manière aussi claire dans la presse francophone qu'anglophone. Si l'on se penche plus précisément sur l'article (ou news), genre étudié dans ce travail, par le biais de manuels de journalisme ${ }^{14}$, on découvre que cette distinction macrostructurelle se 
retrouve à un niveau inférieur, puisqu'il est admis en France que l'article, bien que genre informatif par excellence, puisse contenir un commentaire du journaliste.

Les ouvrages généralistes consultés laissaient également entendre que le droit des médias anglais était plus strict qu'en France, en Suisse et aux États-Unis. Cette remarque a été corroborée par une conférence de presse donnée le 11 août 2006 par le Secrétaire d'État américain à la sécurité intérieure justifiant son refus de divulguer certaines informations à la presse : «[...] because of the rules that govern legal proceedings in Britain, I think we ought to withhold reaching a final conclusion until we've got all the evidence in $»^{15}$. Selon Bertrand (1998) par exemple, la Libel Law britannique est une des lois sur la diffamation les plus strictes du monde occidental, ce qui implique que les journalistes font en sorte de pouvoir prouver tout ce qu'ils avancent. Une autre loi, Contempt of Court, protège les parties en présence dans un procès: ses conditions d'application sont si sévères qu'il devient illégal de faire paraître des informations sur l'accusé ou sur les circonstances de l'affaire en cause qui ne seraient pas avérées par la Cour (Glaser 1998: 110). Considérant ces deux lois, on peut poser l'hypothèse que la presse britannique sera bien plus réticente à divulguer des informations sur les présumés terroristes que la presse américaine, française ou suisse. Dans le cas d'une attaque terroriste, d'autres lois entrent en jeu, notamment Official Secrets Act qui s'applique aux renseignements ayant trait aux forces armées, aux services secrets et aux affaires étrangères (Glaser 1998 : 110). De plus, parallèlement au Patriot Act voté après les attentats de New York aux États-Unis, le Royaume-Uni a adopté des Terrorism Acts successifs (notamment en 2000 et en 2006) ${ }^{16}$. Bertrand évalue l'influence du droit des médias en Grande-Bretagne dans les termes suivants :

Il existe une obsession du secret plus forte que dans le reste de l'Europe, due pour une part à l'absence de séparation des pouvoirs exécutif et législatif. Elle est aggravée par une tradition journalistique de respect pour les autorités. (Bertrand $1998: 108)$

Ces remarques comparatives peuvent aiguiller la réflexion vers l'hypothèse que les journalistes britanniques seront enclins à utiliser plus de précautions oratoires que leurs homologues.

D'ailleurs, les résultats de l'analyse de contenu (cf. tableau 4 en annexe 5) soulignent proportionnellement plus de marques de modalité dans The Guardian que dans les autres journaux (The Sun étant un quotidien populaire, il ne peut être comparé dans les mêmes termes sans prendre en compte une variable supplémentaire ${ }^{17}$. Les catégories définies tentent de couvrir les différentes possibilités grammaticales d'expression de l'hypothèse. Sont regroupés sous «adjectifs, adverbes, substantifs et verbes hypothétiques " les termes de ces classes grammaticales exprimant une incertitude du locuteur par rapport à l'énoncé18. Contrairement à ce qu'affirment certaines grammaires (Thomson \& Martinet 2005), le conditionnel n'existe pas en anglais : « il n'existe pas de marqueur verbal équivalent en anglais» (Celle 2006:3). Il est donc impossible de comparer les sous-catégories de la modalité une à une. La valeur dite «conditionnelle» (Adamczewski 1982: 162) s'exprime donc principalement par les modaux au prétérit. Par conséquent, en anglais, les résultats pour la sous-catégorie " conditionnel » sont nuls tandis que ceux pour les auxiliaires modaux sont largement supérieurs (cf. annexe 5). Néanmoins, le but de ce travail n'est pas d'ouvrir la polémique sur la question du conditionnel en anglais. 
La sous-catégorie "auxiliaires modaux " renvoie aux modal verbs anglais et à leurs équivalents français (pouvoir et devoir). Quant aux "constructions hypothétiques ", elles englobent les subordonnées hypothétiques ou les inversions verbe-sujet suivies du prétérit modal en anglais et du subjectif en français. Une des hypothèses posées se vérifie donc: le quotidien britannique de référence The Guardian est le journal où l'on retrouve le plus d'expressions hypothétiques.

L'analyse de contenu montre également que l'International Herald Tribune et The Guardian devancent largement leurs homologues francophones en termes de discours rapporté (qu'il soit direct ou indirect), avec des fréquences respectivement de $18 \%$ et 12 \%. À titre indicatif, Le Monde reste loin derrière avec 8 \% de discours rapporté.

\subsection{Des représentations divergentes}

La présentation du cadre de réflexion qui soutient les hypothèses de ce travail amène finalement à présenter une partie des résultats finaux. Comme l'affirme Esquenazi (2002: 36), des médias ancrés dans des territoires culturels et linguistiques différents ne peuvent que représenter la réalité à travers le prisme des valeurs et des attentes qui sont liées à ces territoires. Pour cet auteur, il paraît évident que les divergences existant entre deux journaux francophones dans des sociétés radicalement différentes (comme Le Figaro en France et El Watan en Algérie) seront plus grandes qu'entre des journaux en langues différentes dans des sociétés où le mode de vie est plus proche (par exemple entre la Suisse et l'Allemagne).

40 Si l'on aborde les résultats par groupe thématique, on arrive à quelques remarques particulières. L'objectif des attentats, par exemple, reste assez implicite dans la presse britannique. On peut supposer que la couverture médiatique la plus immédiate a été réalisée par la radio et la télévision, préparant ainsi le terrain de la presse écrite, qui n'avait alors plus besoin de contextualiser une nouvelle largement répandue. En France ou en Suisse romande en revanche - et cela peut ouvrir quelques pistes pour la traduction -, l'événement n'a pas été aussi médiatisé qu'outre-Manche, ce qui exige de le situer mieux géographiquement. Les États-Unis, pour leur part ${ }^{19}$, orientent plus le discours sur la menace pour le monde entier.

41 En ce qui concerne le cadrage, les journaux britanniques établissent souvent des liens avec le problème de l'islamisme extrémiste dans leur pays. La France et la Suisse romande renvoient plutôt au contexte du terrorisme et d'al-Qaïda, étant moins concernées par cette question de société. L'International Herald Tribune fait plus allusion à l'Afghanistan, au Pakistan et aux groupes terroristes dans ses articles. Peut-être cette différence s'explique-t-elle par des raisons politiques.

Le discours sécuritaire, quant à lui, fait l'objet d'implications bien tranchées dans les différents journaux. Le Monde, par exemple, fait état de nouvelles technologies toutes plus fantasques les unes que les autres, qui devraient, à l'avenir, améliorer les contrôles de sécurité dans les aéroports. Face à ce discours technique, on trouve les listes d'objets interdits à bord publiées par l'International Herald Tribune, des listes qui rivalisent d'exhaustivité entre elles. En Grande-Bretagne en revanche, on parle relativement peu des mesures prises ou envisagées.

Enfin, les quotidiens de chaque pays représentent différemment les arrestations des suspects. Dans les journaux francophones, on joue plus sur la légende qui auréole les services secrets. Les États-Unis et le Royaume-Uni ne peuvent réagir avec une telle 
distance narrative étant donné que les deux pays ont apparemment mené l'enquête conjointement. Si The Guardian et The Sun dévoilent l'enquête dans sa globalité, avec une rétrospective de ses étapes et une projection sur ses suites, l'International Herald Tribune met plus l'accent sur la collaboration réussie entre les deux partenaires.

En guise de conclusion, il semble nécessaire de rappeler que les informations historiques, géopolitiques, journalistiques ou juridiques évoquées dans cette partie ne sont pas des réponses aux résultats exposés, mais bien au contraire le départ de pistes de réflexion qui mènent à ces résultats, brièvement présentés.

\section{Remarques conclusives}

Cet aperçu des résultats est certes bref, mais il n'y a pas le lieu ici d'entrer dans les détails de l'analyse de discours. Cette étude nourrit plutôt l'ambition d'esquisser quelques lignes directrices utiles au transfert interlinguistique et interculturel comme la question des genres de discours et de leurs variations dans plusieurs communautés linguistico-culturelles différentes :

This means, for example, that the form and genre of the TT [target text] must be guided by what is functionally suitable in the TT culture, rather than by merely copying the ST [source text] profile. (Munday $2001: 77$ )

L'analyse comparative ou contrastive revêt une importance toute particulière dans ce cadre. Pour rappel, la présente étude ne se place pas dans la perspective de traduire des mots, ni même des ensembles de mots, mais des conventions textuelles, d'où l'utilité d'un corpus comparable bilingue ou multilingue. En effet, on ne cherche pas à déterminer les particularités de la « langue » des traducteurs (ce que vise Baker avec les corpus parallèles $)^{20}$, mais à étudier l'usage de la langue par des locuteurs natifs pour en tirer des conclusions utiles à la traduction. Cette approche, qui met en scène le texte comme construction sociolinguistique au carrefour entre un genre et une culture, montre en réalité que peu de différences sont liées au système linguistique uniquement.

Qu'en est-il de la polyphonie dans le cas du corpus analysé ? Tout d'abord, la forte homogénéité des sources transmises par les agences de presse et citées dans les journaux provoque d'inévitables ressemblances entre les articles : les journalistes ont probablement tous eu accès à des informations très similaires. Ainsi, malgré la marge d'incertitude inhérente à l'événement du corpus, les mêmes faits se retrouvent à quelques différences près dans tous les articles. Ensuite, la notion de polyphonie développée dans cet article englobe également la mémoire collective médiatique et, en l'occurrence, les références à une même "famille d'événement»: le terrorisme islamiste post-11 septembre 2001. Les analogies avec des événements de cette famille, et plus particulièrement avec le 11 septembre considéré par certains auteurs comme l'élément déclencheur de ce moment discursif, sont nombreuses dans tous les articles. Enfin, on observe dans tous les articles un cadre d'interprétation clairement occidental avec un Occident laïque posé en victime d'un islam extrémiste. Dans cette opposition binaire, le terroriste apparaît de manière assez uniforme sous les traits d'un jeune musulman issu de la deuxième génération. De même, le grand responsable est présenté comme al-Qaïda ou l'un des groupes radicaux qui y serait rattaché par des liens assez flous. 
Sous cette homogénéité de surface se cachent par ailleurs un grand nombre de spécificités ou d'implicites socioculturels qu'il est nécessaire de connaître pour pouvoir passer d'une communauté linguistico-culturelle à une autre. Premièrement, les quatre pays étudiés n'ont pas vécu les mêmes événements traumatiques, notamment en ce qui concerne les attentats terroristes. Il n'est donc pas étonnant que les allusions aux attentats meurtriers de Londres en 2005 soient plus nombreuses dans la presse britannique, par exemple. Deuxièmement, les pratiques professionnelles du journalisme varient sensiblement entre la tradition anglo-saxonne et la tradition française. La distinction qui serait opérée dans la presse anglophone entre articles de faits et de commentaire n'existerait pas aussi clairement dans la presse de tradition française. À l'échelle de l'article, il est notable que les journaux en français s'autorisent plus d'hypothèses ou de projections dans l'avenir que leurs équivalents en anglais. Troisièmement, le droit des médias peut venir compléter l'étude des pratiques journalistiques. Le fait que le droit des médias qui régit les affaires judiciaires est bien plus strict en Grande-Bretagne n'est pas sans répercussions sur l'écriture journalistique. L'analyse du corpus montre dans l'ensemble que les quotidiens britanniques taisent parfois des informations non vérifiées et qu'ils ont recours à plus de procédés de modalisation que les autres journaux du corpus. En matière de modalité, seules quelques différences sont dues à la non-équivalence des temps et des modes entre les deux langues.

Finalement, malgré bon nombre de traits communs entre les articles du corpus, la polyphonie est gérée de manière différente dans chaque medium. Ce n'est toutefois pas la langue qui est porteuse des différences les plus flagrantes. La représentation discursive d'un même événement sera bien plus influencée par l'emploi d'un genre de discours (et donc également des pratiques professionnelles qui s'y rattachent) ainsi que par la politique, l'histoire et les préoccupations actuelles d'un pays.

\section{BIBLIOGRAPHIE}

Adamczewski, Henri. 1982. Grammaire linguistique de l'anglais. Paris : Armand Colin.

Agnès, Yves. 2002. Manuel de journalisme : écrire pour le journal. Paris : La Découverte.

Arquembourg-Moreau, Jocelyne. 2003. Le Temps des événements médiatiques. Bruxelles : De Boeck.

Baker, Mona. 1998. «Réexplorer la langue de la traduction : une approche par corpus ». Meta XLIII/4, 1-7.

Bardin, Laurence. 1977. L'Analyse de contenu. Paris : Presses universitaires de Paris.

Bertrand, Claude-Jean. 1995. Les Médias aux États-Unis. Paris : Presses universitaires de Paris.

Bertrand, Claude-Jean. 1998. Les Médias en Grande-Bretagne. Paris : Presses universitaires de Paris.

Celle, Agnès. 2006. Temps et modalité : l'anglais, le français et l'allemand en contraste. Berlin et Berne : P. Lang. 
Dayan, Daniel (dir.). 2006. La Terreur spectacle : terrorisme et télévision. Bruxelles : De Boeck.

De Bonville, Jean. 2000. L'Analyse de contenu des médias : de la problématique au traitement statistique. Bruxelles : De Boeck.

Esquenazi, Jean-Pierre. 2002. L'Écriture de l'actualité. Grenoble : Presses universitaires de Grenoble.

Flichy, Patrice (dir.). 1996. Réseaux 75 « Le temps de l'événement I ».

Glaser, Rosette. 1998. La Société britannique à travers la presse populaire. Paris : Ellipses.

Halbwachs, Maurice. [1950] 1997. La Mémoire collective. Paris : Albin Michel.

Hervouët, Loïc. 1979. Écrire pour son lecteur : guide de l'écriture journalistique. Lille : École supérieure de journalisme de Lille.

Keeble, Richard. [1994] 2007. The Newspapers Handbook. Londres : Routledge.

Kenny, Dorothy. 2001. "Corpora in translation studies ». In Baker, M. Routledge Encyclopedia of Translation Studies. Londres : Routledge, 50-53.

Koren, Roselyne. 1996. Les Enjeux éthiques de l'écriture de presse et la mise en mots du terrorisme. Paris : L'Harmattan.

Maingueneau, Dominique. 2007. Analyser les textes de communication. Paris : Armand Colin.

Martin-Lagardette, Jean-Luc. 2000. Le Guide de l'écriture journalistique. Concevoir, rédiger, présenter l'information. Paris : Syros.

McKane, Anna. 2006. Newswriting. Londres, Thousand Oaks, New Dehli : Sage Publications.

Moirand, Sophie. 2007. Le Discours de la presse quotidienne : observer, analyser, comprendre. Paris : Presses universitaires de Paris.

Mouillaud, Maurice et J.-F. Tétu. 1989. Le journal quotidien : événement, mise en page, illustration, titres, citations, faire savoir, faire croire. Lyon : Presses universitaires de Lyon.

Munday, Jeremy, Introducing Translation Studies : Theories and Applications, Londres : Routledge.

Olohan, Maeve. 2004. Introducting Corpora in Translation Studies. New York : Routledge.

Rachman, Stanley J. 1990. Fear and Courage. New York : W. H. Freeman.

Taguieff, Pierre-André. 2006. L'Imaginaire du complot mondial : aspects d'un mythe moderne. Paris : Mille et une nuits.

Thomson, A. J. et A. V. Martinet. 2005. A Practical English Grammar. Oxford : Oxford University Press.

Wagner-Pacifici, E.-R. 2006. « Déchirure symbolique et processus de réparation : les témoins du 11 septembre ». In Dayan, D. (dir.), La terreur spectacle. Terrorisme et télévision. Bruxelles : de Boeck, 211-216.

Weaver, David H. 2007. « Thoughts on agenda setting, framing, and priming ». Journal of Communication 57, 142-147.

Articles du corpus cités dans l'article

Le Monde

Roche, Marc. « Londres déjoue un vaste projet d'attentats contre des avions de ligne ». 11 août 2006. Le Monde. [Mde 11-8-06] 
Roche, M. et P. Smolar. « Le monde en alerte après l'attentat déjoué à Londres ». 12 août 2006. Le Monde. [Mde 12-8-06]

Le Figaro

«Londres : le scénario du complot se précise ». 11 août 2006. Le Figaro. [Fig 11-8-06]

International Herald Tribune

Ward, David. « Britain names terror suspects ». 11 août 2006. International Herald Tribune. [IHT 11-8-06]

The Guardian

Norton-Taylor, R., S. Laville et V. Dodd. " Months of monitoring exposed details of conspiracy ». 11 août 2006. The Guardian. [Guard 11-8-06:1]

Muir, H., J. Vasagar, D. Pallister et D. Campbell. « Arrest of 'normal' neighbours shocks residents ». 11 août 2006. The Guardian. [Guard 11-8-06:2]

The Sun

« Bottle bombers ». 11 août 2006. The Sun. [Sun 11-8-06]

\section{ANNEXES}

\section{Annexe 1}

Tableau 1. Liste des catégories (en gras) et des sous-catégories

\begin{tabular}{|c|c|}
\hline \multicolumn{2}{|l|}{ Catégories et sous-catégories } \\
\hline Terreur & Armes \\
\hline terreur & nom et/ou composition chimique \\
\hline terrorisme, terroriste & bombe ou mot synonyme \\
\hline peur et synonymes & référence à la forme liquide de l'explosif \\
\hline champ lexical de la mort & détails concernant la mise à feu de l'explosif \\
\hline Menace & détails sur les stratégies pour camoufler les explosifs \\
\hline complot & information sur la fabrication de l'explosif \\
\hline attentat, attack & Auteurs \\
\hline risque, alerte et synonymes & $\begin{array}{l}\text { désignation juridiquement acceptable (ex : suspect, } \\
\text { terroriste présumé, etc.) }\end{array}$ \\
\hline élevé, critique, maximal, rouge & désignation « terroriste » \\
\hline sévère, sérieux, orange & nationalité britannique \\
\hline
\end{tabular}




\begin{tabular}{|c|c|}
\hline Interprétations sous-jacentes & origine ethnique / religieuse \\
\hline al-Qaïda & résidence ou interpellation au Royaume-Uni \\
\hline autres groupes extrémistes & comportement garant d'intégration \\
\hline Pakistan (ou lieux situés au Pakistan) & apparence garante d'intégration \\
\hline allusions aux musulmans, à l'islam & conversion à l'islam et modifications du comportement \\
\hline référence à un autre attentat & Modalité \\
\hline Cible & conditionnel \\
\hline Grande-Bretagne & voix passives \\
\hline États-Unis & adjectifs exprimant l'hypothèse \\
\hline monde & adverbes exprimant l'hypothèse \\
\hline avions & substantifs exprimant l'hypothèse \\
\hline Sécurité & verbes exprimant l'hypothèse \\
\hline déjouer et synonymes & modaux \\
\hline arrêter et mots de la même famille & constructions hypothétiques \\
\hline $\begin{array}{l}\text { police, services de sécurité / de } \\
\text { renseignement }\end{array}$ & \\
\hline $\begin{array}{l}\text { phrases qui expriment une coopération } \\
\text { transnationale }\end{array}$ & discours rapporté \\
\hline mesures de sécurité dans les aéroports & \\
\hline $\begin{array}{l}\text { interdictions portant sur les bagages à } \\
\text { main }\end{array}$ & \\
\hline
\end{tabular}

\section{Annexe 2}


Figure 1. Composition du corpus pour l'analyse de contenu : nombre d'articles par jour et par journal

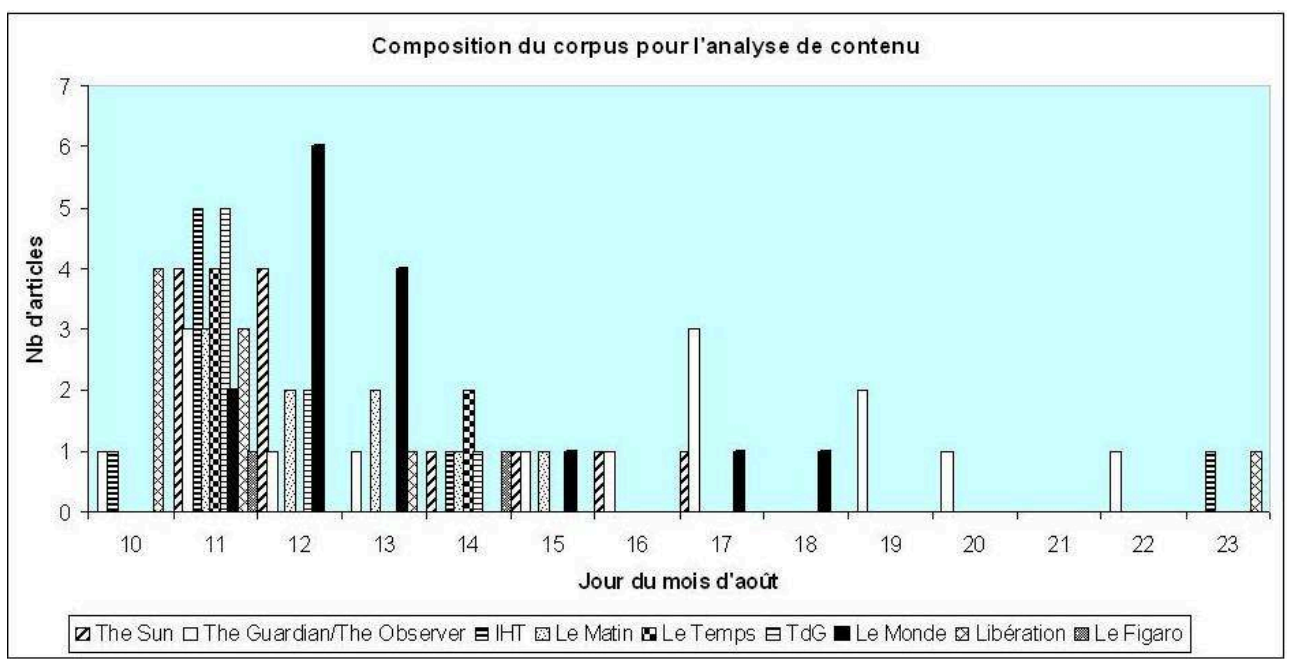

\section{Annexe 3}

Figure 2. Composition du corpus pour l'analyse de discours

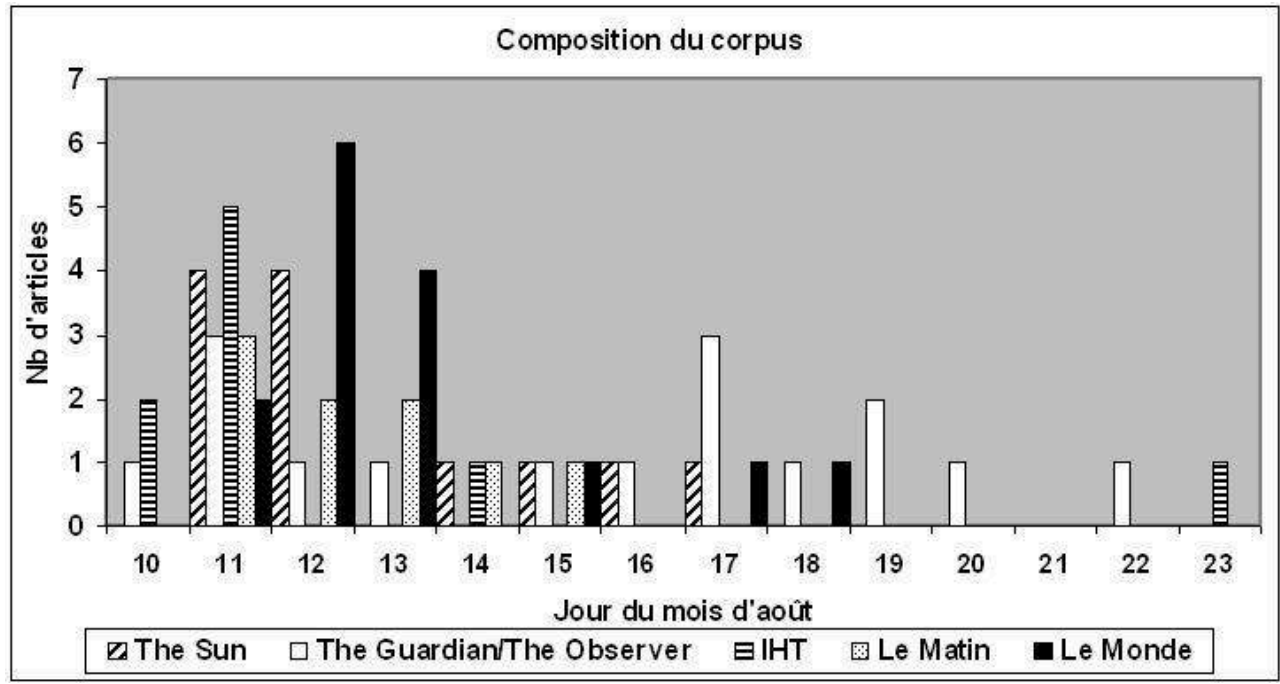

Annexe 4 
Tableau 2. Composition du corpus pour l'analyse de contenu

\begin{tabular}{|c|c|c|c|c|c|c|c|c|c|c|c|c|c|c|c|c|}
\hline \multicolumn{17}{|c|}{ Nombre de mots et d'articles par jour et par quotidien } \\
\hline $\begin{array}{c}\text { Quotidien / Jour du } \\
\text { mois d'août }\end{array}$ & & 10 & 11 & 12 & 13 & 14 & 15 & 16 & 17 & 18 & 19 & 20 & 21 & 22 & 23 & Total \\
\hline \multirow{2}{*}{ The Sun } & art. & & 4 & 4 & & 1 & 1 & 1 & 1 & & & & & & & 12 \\
\hline & mots & & 2228 & 2044 & & 484 & 867 & 329 & 170 & & & & & & & $6^{\prime} 122$ \\
\hline \multirow[t]{2}{*}{$\begin{array}{c}\text { The Guardian/The } \\
\text { Observer }\end{array}$} & art. & 1 & 3 & 1 & 1 & & 1 & 1 & 3 & & 2 & 1 & & 1 & & 15 \\
\hline & mots & 1075 & 3012 & 463 & 922 & & 1136 & 1246 & 2314 & & 2161 & 907 & & 570 & & 13806 \\
\hline \multirow[t]{2}{*}{ IHT } & art. & 1 & 5 & & & 1 & & & & & & & & & 1 & 8 \\
\hline & mots & 716 & 2793 & & & 740 & & & & & & & & & 894 & $5^{\prime} 143$ \\
\hline \multirow[t]{2}{*}{ Le Matin } & art. & & 3 & 2 & 2 & 1 & 1 & & & & & & & & & 9 \\
\hline & mots & & 1073 & 799 & 264 & 303 & 238 & & & & & & & & & $2^{\prime} 677$ \\
\hline \multirow[t]{2}{*}{ Le Temps } & art. & & 4 & & & 2 & & & & & & & & & & 6 \\
\hline & mots & & & & & & & & & & & & & & & $3^{\prime} 273$ \\
\hline \multirow[t]{2}{*}{ TdG } & art. & & 5 & 2 & & 1 & & & & & & & & & & 8 \\
\hline & mots & & & & & & & & & & & & & & & 3326 \\
\hline \multirow[t]{2}{*}{ Le Monde } & art. & & 2 & 6 & 4 & & 1 & & 1 & 1 & & & & & & 15 \\
\hline & mots & & 1165 & 3210 & 2359 & & 465 & & 569 & 737 & & & & & & 8505 \\
\hline \multirow{2}{*}{ Libération } & art. & 4 & 3 & & 1 & & & & & & & & & & 1 & 9 \\
\hline & mots & 1646 & 1612 & & 200 & & & & & & & & & & 531 & $3^{\prime} 989$ \\
\hline \multirow[t]{2}{*}{ Le Figaro } & art. & & 1 & & & 1 & & & & & & & & & & 2 \\
\hline & mots & & 1064 & & & 248 & & & & & & & & & & 1'312 \\
\hline & & & & & & & & & & & & & & & & \\
\hline $\mathrm{Nb}$ d'articles EN & & & & & & & & & & & & & & & & 35 \\
\hline $\mathrm{Nb}$ de mots EN & & & & & & & & & & & & & & & & 25071 \\
\hline Nb d'articles FR & & & & & & & & & & & & & & & & 49 \\
\hline Nb de mots FR & & & & & & & & & & & & & & & & 23082 \\
\hline Nb d'articles total & & & & & & & & & & & & & & & & 84 \\
\hline $\mathrm{Nb}$ de mots total & & & & & & & & & & & & & & & & $48 ' 153$ \\
\hline
\end{tabular}

\section{Annexe 5}

Tableau 3. Fréquence d'apparition des marques de modalité

\begin{tabular}{|l|l|l|l|l|l|l|}
\hline \multicolumn{7}{|l|}{ Fréquence d'apparition des marques de modalité en \%o } \\
\hline catégorie & quotidien & IHT & Guard. & Sun & Monde & Matin \\
\hline adjectifs hypoth. & 1,4 & 3,5 & 1 & 2 & 4 \\
\hline adverbes hypoth. & 2 & 2 & 1,6 & 1,6 & 1 \\
\hline substantifs hypoth. & 0,9 & 2,3 & 1 & 1 & 0,7 \\
\hline verbes hypoth. & 1,7 & 3,1 & 1,7 & 2 & 1 \\
\hline conditionnel & 0 & 0 & 0 & 5,2 & 3,7 \\
\hline auxiliaires modaux & 11,5 & 15,8 & 18,4 & 3 & 5 \\
\hline constructions hypoth. & 1,2 & 2,5 & 2 & 1,1 & 0,7 \\
\hline
\end{tabular}

\section{NOTES}

1. Maingueneau (2007: 38) en donne une définition sous forme d'exemple : «Ainsi le talk-show constitue-t-il un genre de discours à l'intérieur du type de discours télévisuel, lui-même partie prenante d'un ensemble plus vaste que serait le type de discours médiatique où figureraient aussi le type de discours radiophonique et celui de la presse écrite ».

2. Maingueneau (2007: 38) décrit les genres de discours comme «des dispositifs de communication qui ne peuvent apparaître que si certaines conditions sociohistoriques sont réunies ». Pour lui, il est donc possible de "caractériser une société par les genres de discours qu'elle rend possibles et qui la rendent possible ».

3. Maingueneau définit la polyphonie en ces termes : "Quand il parle, un locuteur ne se contente pas d'exprimer ses propres opinions, il fait constamment entendre diverses autres voix, plus ou moins clairement identifiées, par rapport auxquelles il se situe ». (2007:110) 
4. «L'analyse de contenu figure parmi les rares procédés mis au point expressément pour l'étude des faits de communication [...]». (de Bonville $2000: 9$ )

5. Centre de recherche sur les discours ordinaires et spécialisés, Université Paris 3 - Sorbonne Nouvelle, 24-04-08 <http://www.cavi.univ-paris3.fr/ilpga/syled/cediscor.htm>.

6. La notion d'«événement» fait l'objet d'une abondante recherche en sciences de la communication et des médias. Voir notamment Arquembourg-Moreau (2003) ou Flichy (1996).

7. Cf. terminologie employée par Mouillaud et Tétu (1989).

8. Pour des raisons techniques, il n'a pas été possible d'accéder aux archives du New York Times. Or on peut considérer l'International Herald Tribune comme l'édition internationale du Washington Post et du New York Times. Certes, comme son nom l'indique, l'International Herald Tribune s'adresse surtout à un lectorat hors des États-Unis, mais il n'en reste pas moins un journal américain. Il s'agit donc d'un pis-aller.

9. Quotidien de référence en Suisse romande, 24-04-08<Erreur ! Référence de lien hypertexte non valide.

10. Quotidien local de référence publié à Genève, 24-04-08 < Erreur! Référence de lien hypertexte non valide.

11. C'est le cas en ce qui concerne les affirmations du Président des États-Unis, George W. Bush, sur l'existence d'armes de destruction massive en Irak.

12. Viltard, Yves, "Le cas McCarthy. Une construction politique et savante ", Cultures et Conflits 43, 2001, [en ligne] <http://www.conflits.org/index860.html> (mis en ligne le 28/02/03; dernière consultation le 16/04/08).

13. Mis à part l'exception notable de Pearl Harbor, en 1941. Il faut toutefois préciser que Pearl Harbor était une base militaire située dans l'archipel d'Hawaï, soit à environ $4000 \mathrm{~km}$ de la côte ouest des États-Unis.

14. Voir entre autres Martin-Lagardette 2000, Agnès 2002, Hervouët 1979, Keeble 2007 et McKane 2006.

15. «Press Conference with Secretary of Homeland Security Michael Chertoff and Assistant Secretary for TSA Kip Hawley », 11 août 2006. 28-04-08 <http://www.dhs.gov/xnews/releases/pr_ 1158350768683.shtm>.

16. Cf. Office of Public Sector Information, 18 avril 2008. 22-04-08 <http://www.opsi.gov.uk/>.

17. Il est nécessaire de préciser que les chiffres obtenus sont une fréquence exprimée en \%o (nombre d'occurrences par rapport au nombre total de mots).

18. Par exemple « apparent " pour les adjectifs, « likely» pour les adverbes, " possibility» pour les substantifs, et « to believe » pour les verbes.

19. Dans cette section, les noms de pays renvoient par métonymie aux journalistes des quotidiens étudiés dans le pays en question.

20. « $\mathrm{L}[\mathrm{e}]$ but est d'identifier les traits qui distingueraient la langue anglaise de la traduction de celle des usagers ordinaires ». (Baker $1998: 2$ )

\section{RÉSUMÉS}

Le discours journalistique est le lieu privilégié de la polyphonie et de l'expression culturelle. La présente communication se propose d'explorer les traces laissées par un genre spécialisé (l'article de presse) et par des imaginaires collectifs dans la presse francophone et anglophone. 
Un corpus bilingue est soumis à une analyse de contenu, puis à une analyse de discours. Ensuite est examiné le rapport dialogique qu'entretiennent les articles avec leurs sources. Dans le cas de ce corpus, l'homogénéité et le nombre limité de sources induit d'incontournables similitudes dans le contenu des articles. On part en outre du postulat que la mémoire collective (occidentale) du terrorisme construite par les médias rapproche les discours journalistiques de différents pays. Par ailleurs, il est important de se demander dans quelle mesure les différences entre l'histoire, le contexte sociopolitique et les pratiques journalistiques de chaque pays peuvent influencer la représentation d'un même événement. S'appuyant sur un corpus comparable bilingue (donc sans traductions), la méthode comparative permet d'étudier les pratiques textuelles communes à un type de discours précis, ou spécifiques à une communauté culturelle.

The language of the press is a field in which polyphony and cultural expression are particularly important. This paper investigates the traces of a specialised genre (news) and of collective memory in English-language and French-language daily newspapers. For this purpose, a bilingual corpus is analysed using content analysis and discourse analysis methods.

The relation between the sources and the media production is also thoroughly examined. In this particular case, the homogeneity and the small number of sources necessarily induce similarities in the articles' content. This reflection is also based on the assumption that the media has constructed a (Western) collective memory of terrorism which should be shared by the analysed countries. On the other hand, it is important to observe to what extent history, the socio-political context and journalistic practices can influence the representation of the same event in several countries. This comparative analysis is based on a bilingual comparable corpus (i.e., not on translated texts), which makes it possible to explore similarities and differences due to genre and cultural uses.

\section{INDEX}

Mots-clés : analyse contrastive, analyse du discours, genre textuel, presse, terrorisme Keywords : contrastive analysis, discourse analysis, press, terrorism, textual genre

\section{AUTEUR}

\section{LUCILE DAVIER}

Lucile Davier est assistante d'enseignement et de recherche au Département français de traductologie et de traduction de l'École de traduction de Genève. Lucile.Davier@unige.ch 